\title{
Long-Term Increases in Dentate Granule Cell Responsivity Accompany Operant Conditioning
}

\author{
R. W. Skelton, A. S. Scarth, D. M. Wilkie, J. J. Miller, ${ }^{1}$ and A. G. Phillips \\ Departments of Psychology and 'Physiology, University of British Columbia, Vancouver, Canada, V6T $1 Y 7$
}

\begin{abstract}
The efficacy of synaptic transmission from the perforant path (PP) to the granule cells in the dentate gyrus (DG) of freely moving rats was monitored electrophysiologically over the course of training in an appetitively motivated, discriminated operant paradigm. Every day, $22 \mathrm{hr}$ after behavioral sessions, evoked potentials were recorded from the DG following stimulation of the PP over range of current intensities and the amplitudes of the population spikes were measured. Behavioral conditions involved training in an operant conditioning paradigm or a session of free-feeding. Significant increases in population spike amplitudes were observed over the $8 \mathrm{~d}$ of training, but not over the $8 \mathrm{~d}$ of free-feeding. This training-induced increase in granule cell responsivity persisted for at least $10 \mathrm{~d}$ following the cessation of behavioral trials and was in many ways comparable to long-term potentiation (LTP), subsequently observed in these same rats $24 \mathrm{hr}$ after tetanic stimulation. These data confirm and extend previous reports of synaptic enhancements following conditioning and suggest that such increases in synaptic efficacy may encode some aspect of learning.
\end{abstract}

Studies of neuroplasticity have identified several important phenomena of immediate relevance to the analysis of neural substrates of learning and memory (Lynch et al., 1978). One particularly striking form of plasticity is long-term potentiation (LTP), a process by which the efficacy of synaptic connections can be increased significantly by relatively brief periods of highfrequency stimulation (Bliss and Gardner-Medwin, 1973; Douglas and Goddard, 1975; Dolphin, 1985). Support for the involvement of LTP-related mechanisms in learning and memory comes from its cnduring stability, its induction by normal patterns of electrical activity, its occurrence in brain regions such as the hippocampus, previously linked to learning/memory processes, and, most recently, from the observation of synaptic enhancement following learning (Lynch and Baudry, 1984; Morris and Baker, 1984; Teyler and DiScenna, 1984).

To date, 2 strategies have been used to evaluate the relation between changes in synaptic efficacy and memories of biologically significant events (Morris and Baker, 1984). The induction of LTP by tetanic stimulation and subsequent observation of changes in learning rates is referred to as "prior enhancement." "Prior learning" involves training animals in a behavioral par-

\footnotetext{
Received Aug. 8, 1986; revised Apr. 27, 1987; accepted Apr. 27, 1987.

This work was supported by Grant 7808 from the Natural Sciences and Engineering Research Council of Canada to A.G.P.

Correspondence should be addressed to Dr. R. W. Skelton at his present address: Department of Psychology, University of Victoria, P.O. Box 1700, Victoria, B.C. Canada, V8W 2 Y2.

Copyright @ 1987 Society for Neuroscience $0270-6474 / 87 / 103081-07 \$ 02.00 / 0$
}

adigm and subsequent measurement of stimulation-evoked potentials to reveal training-induced changes in synaptic efficacy.

There have been 3 reports on the effects of "prior enhancement," 2 of which directly indicate the contribution of increased synaptic efficacy to the acquisition of new behavioral responses. In the first, induction of LTP was shown to facilitate discriminative classical conditioning of the nictitating membrane response in rabbits (Berger, 1984). In the second, LTP was shown to improve behavioral detection of low-intensity perforant pathway (PP) stimulation and accelerate subsequent acquisition of stimulus control by this brain stimulation in an appetitively motivated operant paradigm (Skelton et al., 1985). In the third, LTP disrupted acquisition of new spatial information, but did not alter well-established spatial memories (McNaughton et al., 1986). This last effect could have been the result of LTP's exhausting the entire population of modifiable synapses, as the authors suggest, or alternatively, it could have been due to LTP's strengthening of existing spatial memories to the point where they could not be modified by altered spatial relations. In either case, this last study, like the first 2 , clearly indicates a relation between increased synaptic efficacy and learning.

The alternative procedure of "prior learning" has also provided evidence for a role of increased synaptic efficacy in learning. Increases in population spike amplitudes in the hilus of the dentate gyrus have been observed (1) during sessions of classical conditioning of the nictitating membrane response (Weisz et al., 1984), (2) 4 and $24 \mathrm{hr}$ after a block of trials in a one-way avoidance paradigm, with tones or PP stimulation as the conditional stimulus (Rüthrich et al., 1982; Matthies et al., 1986), (3) for several days after the onset of exposure to a novel, complcx environment (Sharp ct al., 1985), and (4) after 3-4 wceks of rearing in a complex environment (Green and Greenough, 1986). In most of these studies, the practice of evaluating synaptic efficacy through measurement of extracellular field potentials from the hilus of the dentate gyrus (DG) makes it appropriate to use the term "synaptic communication," rather than "synaptic efficacy." Synaptic communication is meant to include all factors that modulate postsynaptic responses to afferent input, including synaptic efficacy, postsynaptic thresholds, and tonic afferent inputs. Changes in population spike amplitudes cannot distinguish between the contributions of these factors.

The question of whether increased synaptic communication follows appetitive learning remains unanswered and the present study addresses this issue. Electrophysiological recordings of DG population spikes evoked by PP stimulation were made outside of the context of the learning paradigm. In this manner, any observed changes in synaptic communication could be dissociated from specific stimuli or motor responses accompanying discriminated operant conditioning. Long-term monitoring of synaptic communication across control and experimental phas- 


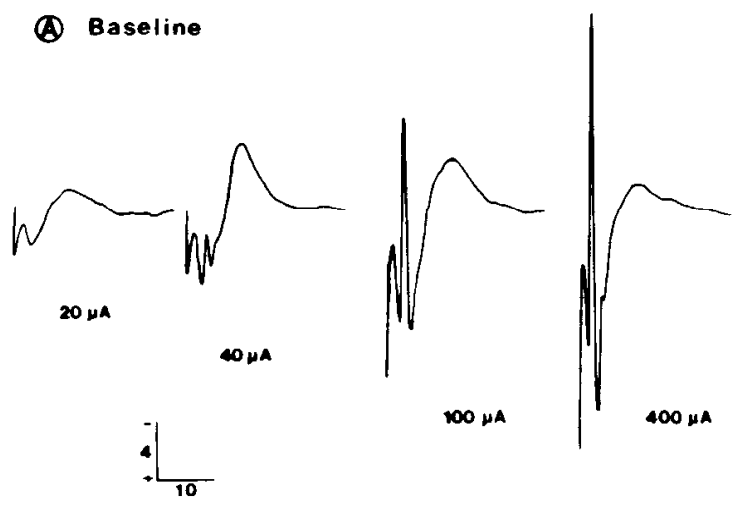

(c)

Conditioning Day 5

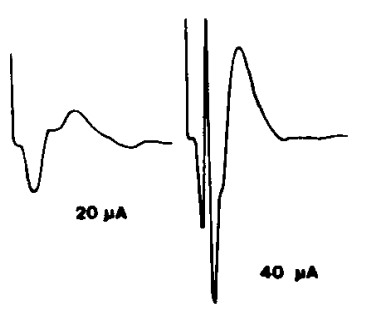

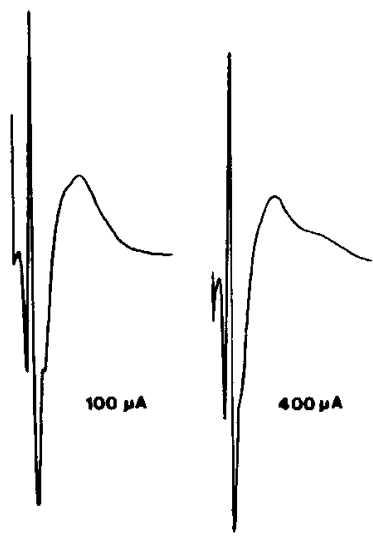

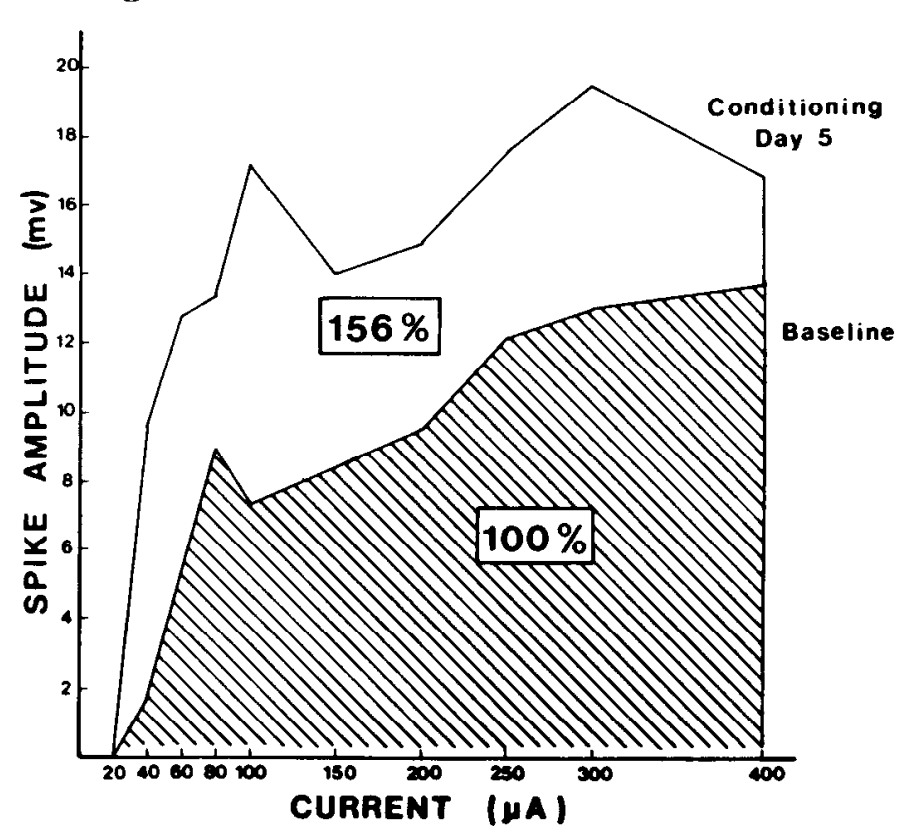

Figure 1. Transformation of evoked potential data. Sample evoked potentials taken from one rat on $(A)$ the baseline day and $(B)$ conditioning day 5. $C$, Amplitudes of population spikes from these 2 days are plotted against current intensity, and the area under each curve is calculated geometrically. The areas of all curves are compared to the baseline area (shaded) by calculating a percentage.

es of the study was also incorporated to determine the duration and persistence of any changes associated with learning. Upon completion of behavioral testing, all subjects were screened for LTP induced by tetanic stimulation of the PP, thereby providing direct comparisons between any training-induced potentiation and LTP.

\section{Materials and Methods}

Surgery. All methods used for surgery and electrode preparation were very similar to those reported previously (Skelton et al., 1985). Briefly, each of 28 male hooded rats weighing $350-450 \mathrm{gm}$ was anesthetized with sodium pentobarbital $(60 \mathrm{mg} / \mathrm{kg})$ and implanted with a monopolar, extracellular recording electrode in the hilus of the left DG and a monopolar stimulating electrode in the left PP. Recording electrodes were 62 $\mu \mathrm{m}$ stainless steel wire, Teflon-coated to the flush-cut tips, and were targeted to the following stereotaxic coordinates: $3.4 \mathrm{~mm}$ posterior to bregma, $1.8 \mathrm{~mm}$ lateral to the midline, and $3.1 \mathrm{~mm}$ ventral to dura. Stimulating electrodes were No. 00 stainless steel insect pins insulated to within $0.4 \mathrm{~mm}$ of the tip and were targeted to $8.0 \mathrm{~mm}$ posterior to bregma, $4.5 \mathrm{~mm}$ lateral to the midline, and $2.5 \mathrm{~mm}$ ventral to dura. Constant-current cathodal pulses $(0.1 \mathrm{msec}, 0.2 \mathrm{~Hz}, 500 \mu \mathrm{A})$ were delivered to the PP during surgery, and the resulting evoked potentials were used to guide the final placement of both electrodes. The recording electrode was positioned for a maximum-amplitude, negative-going (i.e., hilar) population spike, and the stimulating electrode was positioned such that the threshold of the population spike was less than $100 \mu \mathrm{A}$. Current-return and indifferent-recording electrodes were derived from separate sets of skull screws. All electrodes were soldered to Amphenol connector pins and the entire assembly was fixed in place with dental acrylic.

After a recovery period of at least a week, each rat was given a single pulse of stimulation at $250 \mu \mathrm{A}$, and the resulting evoked potentials were used to select the 12 subjects with the largest hilar population spikes. These rats were food-deprived to $85 \%$ body weight gradually over a 3 week period, and then behavioral and electrophysiological testing was begun.

After completion of all testing, the rats were given an overdose of urethane and perfused with saline and formalin. The brains were removed for standard histological confirmation of the electrode placements.

Electrophysiological recordings. This method was decribed in detail previously (Skelton et al., 1983). Briefly, each subject was tested daily by measuring the amplitude of the population spikes at 10 different current intensities, ranging from 20 to $400 \mu \mathrm{A}$. Three pulses were presented at each intensity, the intensities were presented in ascending order, and these 30 pulses were presented in a continuous train at 0.04 $\mathrm{Hz}$. The evoked potentials were passed through a 6-channel commutator and a JFET preamplifier (AC-coupled, $10 \mathrm{M} \Omega$, gain $=1.0$ ), filtered $(0.1-$ $3 \mathrm{kHz}$ ), and amplified by a Tektronix storage oscilloscope, then digitally recorded using a Digital 11/03 minicomputer that sampled at $20 \mathrm{kHz}$. Subsequently, the 3 evoked potentials collected at each intensity were averaged together and the population spike amplitude was measured as the voltage difference between the maximum positivity and the maximum negativity of the spike. Input/output (I/O) curves were constructcd for each subject each day by plotting the 10 population spike amplitudes against the 10 current intensities. Each curve was then reduced to single number by calculating the area between it and the $x$-axis, using a geometric formula (Durrant et al., 1961). This process of reducing a set of population spike amplitudes to a percentage based on the areas of $\mathrm{I} / \mathrm{O}$ curves is illustrated in Figure 1.

All subjects were given 3 tests (in 3 d) to habituate them to the electrophysiological procedures before the behavioral sessions were begun. These behavioral manipulations were presented in 2 consecutive "phases," with each phase lasting $8 \mathrm{~d}$ (see below for details). The I/O curve collected on the last day of habituation provided the baseline for Phase 1 , and the I/O curve collected on the last day of Phase 1 provided the baseline for Phase 2 . 
The electrophysiological tests in this experiment were designed to monitor long-term changes in synaptic communication in the DG as related to the acquisition and retention of a discriminated operant response. In order to ensure that any observed changes in efficacy at the PP-DG synapses were not attributable to contextual cues, behavioral arousal, and specific patterns of motor behavior, all recording tests were conducted $22 \mathrm{hr}$ after each daily behavioral session in a test apparatus distinct from the one used for behavioral testing. Specifically, the chambers used for the measurements of the evoked potentials were Plexiglas boxes (30 $\times 24 \times 42 \mathrm{~cm}$ high) with smooth sides, a smooth top, and a wire-mesh floor. This chamber was contained within a dimly lit wooden box $(60 \times 60 \times 60 \mathrm{~cm})$, and the door of this box was left open to allow continuous observation of the subjects. In contrast, the chambers used for behavioral testing were smaller, had brass-rod floors, and were contained in a brightly lit, noisy enclosure (see below for details). The rats were returned to their home cages for the $90 \mathrm{~min}$ interval between the electrophysiological tests and the behavioral trials.

Behavioral methods. The 12 rats that met the electrophysiological criteria were divided randomly into 2 groups and tested in counterbalanced order in 2 behavioral conditions: free-feeding and learning. Group 1 was exposed to the learning paradigm in Phase $1(8 \mathrm{~d})$, while group 2 served as the free-feeding controls. The behavioral conditions were reversed in Phase 2 ( $8 \mathrm{~d})$.

In the free-feeding condition, the rats were given $30 \mathrm{~min}$ to retrieve 40 food pellets (Noyes; $45 \mathrm{mg}$ ) previously placed into a brass food cup protruding from the side of a cardboard box. This box was covered with hardware cloth and maintained in a room illuminated by natural light. In this condition there was no contingency between responding and reward availability. The free-feeding condition therefore served as a control for the effects of the handling and feeding inherent in the learning paradigm, as well as a control for any electrophysiological effects of the I/O curve testing.

The chambers used for the learning condition were distinct in several respects from those used for the electrophysiological tests. They were also constructed from Plexiglas, but were smaller $(24 \times 18 \times 24 \mathrm{~cm}$ high) and had a brass-rod floor. In addition, the doors of the soundattenuating enclosures (wooden boxes, as before) were closed, exposing the subjects to bright illumination and the noisc of the ventilation fan.

Daily sessions in the learning condition consisted of 40 trials presented on a variable-interval $2 \mathrm{~min}$ schedule. On each trial the sequence of events was as follows: a $10 \mathrm{sec}$ baseline period, a $0.1 \sec$ tone $(2.8 \mathrm{kHz})$, a $10 \mathrm{sec}$ response period, and, finally, the delivery of a food pellet (Noyes; $45 \mathrm{mg}$ ) to a brass food cup protruding into the test chamber. An electronic sensor was attached to each food cup such that contacts made by the rats on the rim were registered as responses. Any such contact during the response period led to the immediate delivery of the food pellet for that trial; otherwise, the delivery of the pellet was delayed until the end of the $10 \mathrm{sec}$ response period. Thus, the tone was both a conditional stimulus for food pellet delivery and a discriminative stimulus (DS) signaling a period in which a response was reinforced. Although there was no contingency between responses (cup contacts) and food availability, there was a contingency between responding and the immediacy of reward. In this paradigm, responses that initially resulted from random searches of the food cup were shaped into precise, shortlatency responses for food, confined to the brief period after each DS.

In order to assess the degree of stimulus control exerted by the DS, a discrimination ratio was calculated for each trial and averaged for each session in the following manner: The DS on each trial defined 2 periods, one $(A)$ extending forward to the onset of the first response after the DS ( $10 \mathrm{sec}$ maximum), the other $(B)$ extending backward to the offset of the last response preceding the DS. The discrimination ratio was calculated as $1-[A /(A+B)]$. This ratio fluctuated around 0.50 when responses were random with respect to the DS and rose towards 1.00 as learning progressed. A criterion value of 0.70 was selected because, at this level, responses were almost entirely absent from the preDS period and usually followed the DS within a second. All stimulus contingencies and response measurements were controlled by a Nova 3/12 minicomputer.

Tetanic stimulation. Ten days after the completion of the behavioral manipulations, the rats were tested electrophysiologically for persistence of changes observed during behavioral testing and for residual ability to undergo LTP. After an L/O curve was collected, tetanic stimulation was administered, consisting of 50 trains of 10 pulses, each at $400 \mu \mathrm{A}$. The frequency within the trains was $200 \mathrm{~Hz}$ and the trains were separated by $25 \mathrm{sec}$. The next day, a post-tetanic I/O curve was collected and the change from the pretetanic level was evaluated.

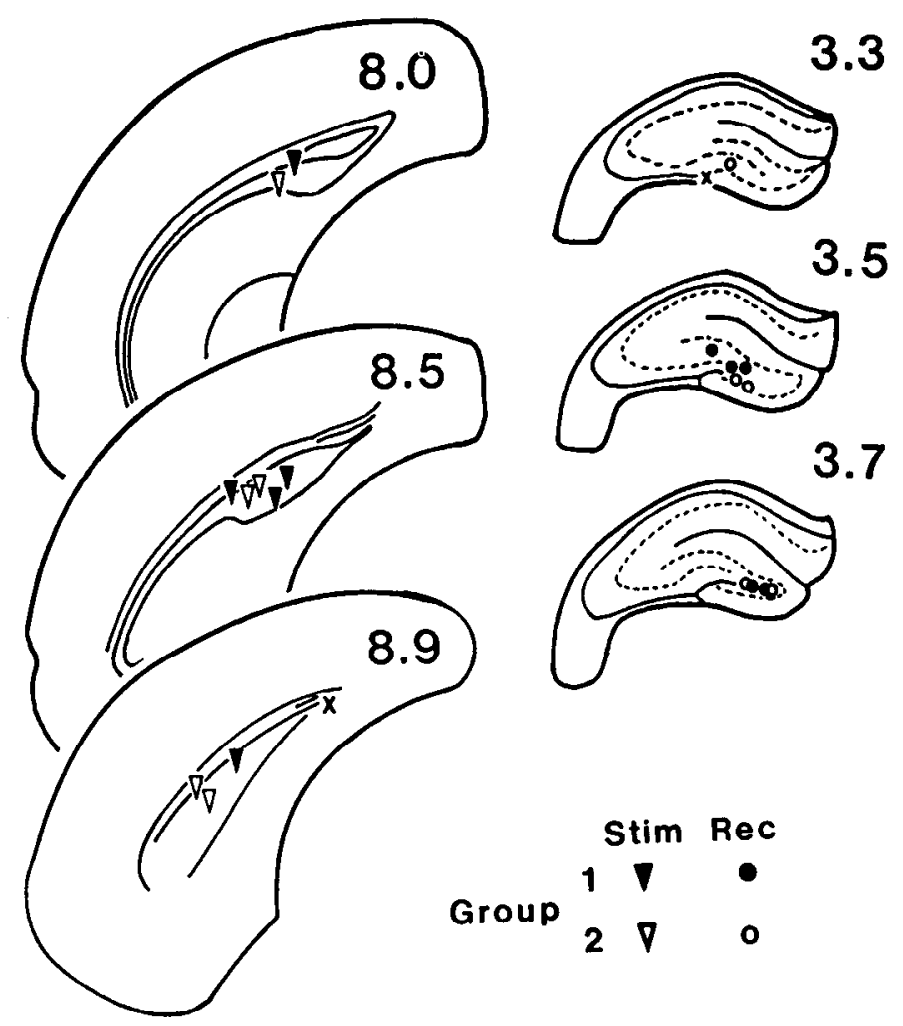

Figure 2. Locations of stimulating (triangles) and recording (circles) clcctrodes for each subject are depicted on coronal sections of the posterior cortex and hippocampus, respectively: Group 1, closed symbols; group 2, open symbols. The numbers indicate the distance (in $\mathrm{mm}$ ) of each partial coronal section from bregma. The $\times$ 's indicate locations of misplaced electrodes that led to the rejection of 2 rats.

\section{Results}

\section{Electrode placements}

In 10 of the 12 rats, the stimulating electrodes were placed in the angular bundle and the recording electrodes were placed in either the hilus or the granule cell layer of the DG (Fig. 2). These placements were consistent with the waveforms of the evoked potentials, all of which contained negative-going population spikes, with peaks occurring at latencies shorter than $5 \mathrm{msec}$. The data from one subject in each group were excluded because the double-population spikes present at low intensities prevented consistent analysis of population spike amplitudes. Histological localization of the electrode placements in these 2 rats confirmed their misplacements: one stimulating electrode was atypically dorsal, thereby producing equivalent activation of both the lateral and medial perforant paths (McNaughton and Barnes, 1977); one recording electrode was located just lateral to the lower blade of the DG, in a position from which doublepopulation spikes are often recorded (personal observation).

\section{Behavior}

All subjects learned the discriminated operant response within $7 \mathrm{~d}$ (Fig. 3). A repeated-measures ANOVA confirmed the significance of the changes in the discrimination ratios over days $(F(7,56)=35.1, p<0.001)$ and revealed no significant effect of the order in which the behavioral conditions were presented. Separate analyses of the data from the 2 groups revealed significant increases in the discrimination ratios for both groups (group 1: $F(7,28)=23.6, p<0.001$; group $2: F(7,28)=13.6$, 


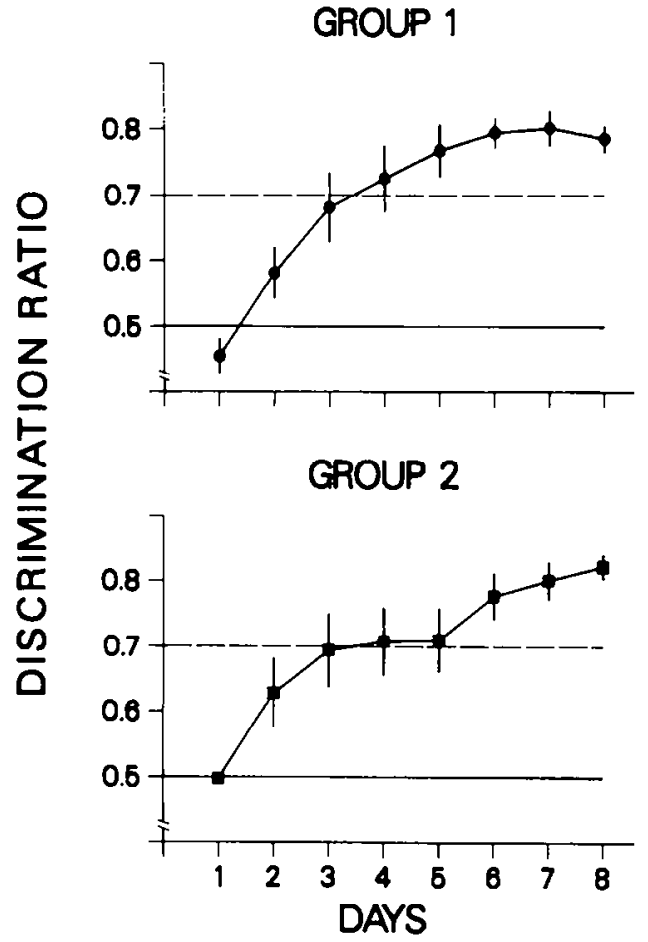

Figure 3. Discrimination ratios (means and SEM, $n$ 's $=5$ ) from the operant paradigm are plotted for each group over days in the learning phase. The criterion for learning was 3 consecutive $d$ with a ratio greater than 0.70 .

$p<0.001)$. The number of days required to reach the criterion ratio of 0.70 ranged from 3 to $6 \mathrm{~d}$ in group 1 (mean, 4.2 ), and from 2 to $7 \mathrm{~d}$ in group 2 (mean, 4.2). Behavior at criterion levels typically consisted of sitting beside the hopper until the DS sounded and then immediately placing a paw on the hopper to obtain a food pellet. Under the free-feeding condition, the subjects typically consumed all 40 food pellets within the first 5 $\min$.

\section{Electrophysiology}

Important differences were observed between the changes in the I/O curves associated with the 2 behavioral conditions (Fig. 4). The population spike amplitudes grew progressively larger under the learning condition, but remained stable or decreased slightly under the free-feeding condition. Statistical analysis of the areas under the I/O curves (as percentages of baseline) confirmed the overall difference between the 2 conditions (repeatedmeasures ANOVA: $F(1,8)=23.19, p<0.002)$ and the difference between the conditions in the change over days $(F(8,64)=3.47, p<0.003)$. No other factors in the analysis were significant, including the order in which the behavioral conditions were presented.

Comparisons of population spike amplitudes over the transitions between behavioral conditions and over the intervals from the end of training to the retest provided valuable new information about the persistence and stability of the traininginduced elevations. For example, increases in the $\mathrm{I} / \mathrm{O}$ curve areas of group 1 that developed during learning did not persist when the behavioral conditions were switched to free-feeding. Rather, the $\mathrm{I} / \mathrm{O}$ curve areas returned to initial baseline levels by the third day of Phase 2 . This decrement did not appear to be a passive dissipation of the earlier increase, because a mea-

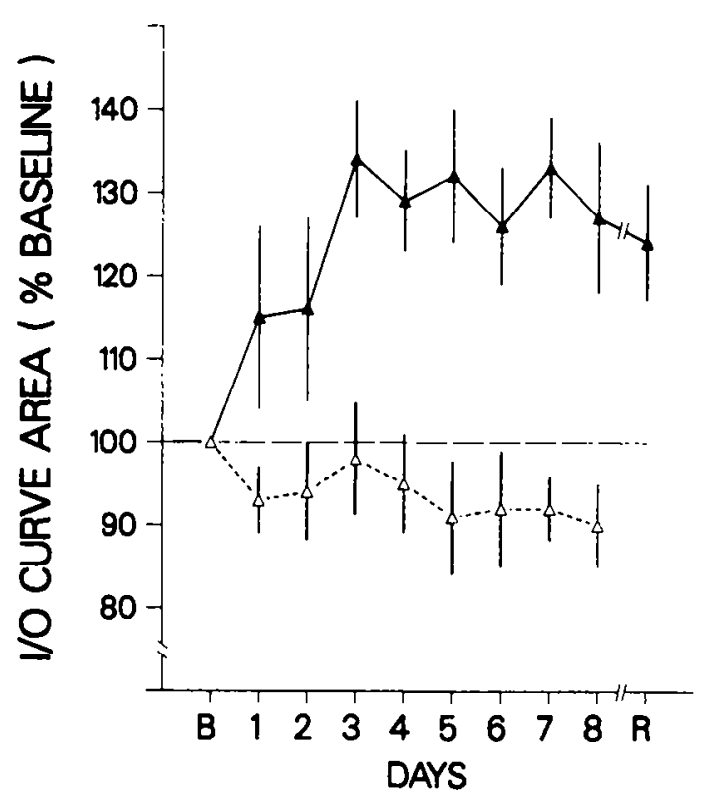

Figure 4. Pcrcentage areas of I/O curves (mean and SEM, $n=10$ ) are shown for the learning condition (closed triangles) and the free-feeding condition (open triangles), collapsed across the 2 groups. Baseline $(B)$ was the last I/O curve taken before the first session in each behavioral condition. The baseline area of the "learning" condition was used to calculate the percentage area on the retest $(R)$.

surement of population spike amplitudes taken $10 \mathrm{~d}$ after the conclusion of behavioral testing revealed amplitudes comparable to those observed following training. Furthermore, little decrement was seen when the training was followed only by the $10 \mathrm{~d}$ rest period in group 2 . An indication of the magnitude of the "savings" between the end of training and the retest, the ratio of $\mathrm{I} / \mathrm{O}$ curve areas, revealed a savings of $1.13 \pm 0.23$ in group 1 and of $0.96 \pm 0.10$ in group 2 (see Table 1). This persistence is also evident in Figure 4 , in which the average I/O curve area was $127.0 \pm 9.0 \%$ of baseline after the last day of learning and $125.1 \pm 8.0 \%$ on the retest.

The final electrophysiological measurements confirmed the capacity of these same PP-DG synapses to undergo LTP. Twenty-four hours after the tetanic stimulation, the areas of the $\mathrm{I} / \mathrm{O}$ curves of every rat were at least $120 \%$ of the pretetanic levels. The mean was $162.5 \%( \pm 13.1)$, and was significantly different from the pretetanic levels $(t(8)=3.35, p<0.01$ ) for 9 rats (the data from one subject were lost). The means for groups 1 and 2 were 161.1 and $164.4 \%$, respectively, and both were different from pretetanic levels $(p<0.05)$.

Examples of $\mathrm{I} / \mathrm{O}$ curves collected in each portion of this experiment, taken from one subject in each group, are presented in Figure 5, which illustrates the similarities between the changes in the $\mathrm{I} / \mathrm{O}$ curves produced by electrical stimulation and by training. Both manipulations reduced population spike threshold in some rats but not in others, and both increased population spike amplitudes over the entire range of intensities tested. The only notable difference was a tendency, described earlier, for tetanic stimulation to produce greater increases in population spike amplitudes.

\section{Discussion}

The present results demonstrate that appetitive operant conditioning can induce a potentiation of synaptic communication similar in many respects to LTP induced by tetanic stimulation. 

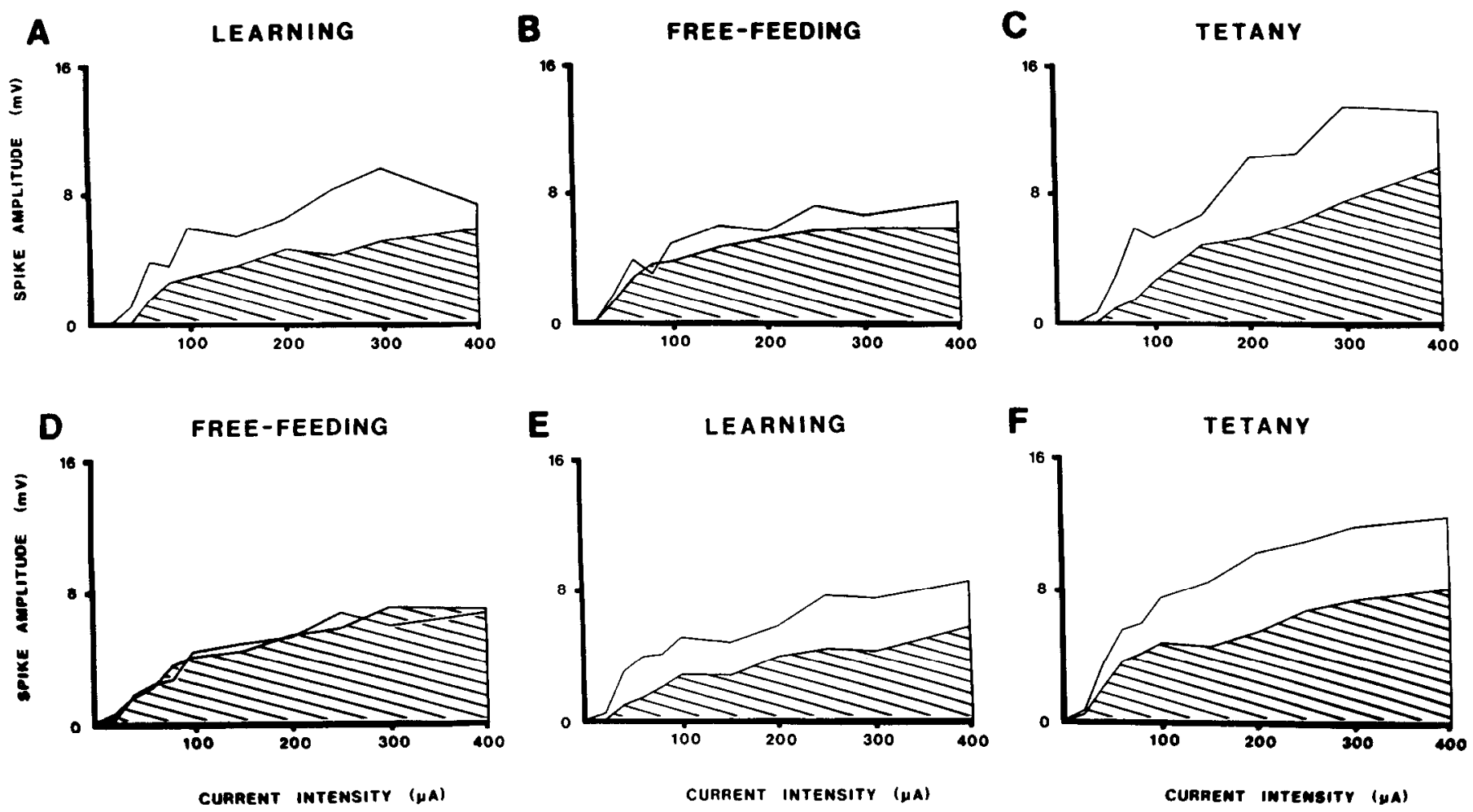

Figure 5. Individual I/O curves from the baseline day (shaded) and one other day in each phase of the experiment are shown for one rat in group $1(A-C)$ and one rat in group $2(D-E)$. (Curves with the largest area in each phase are shown.)

Both behavioral training of an operant response for food and high-frequency stimulation produced increases in population spike amplitudes in the DG. These changes were observed over a large range of current intensities and persisted for more than $22 \mathrm{hr}$. No potentiation was produced by a control condition that included food deprivation, handling, scheduled feeding, and electrophysiological tests equivalent to the training condition. Furthermore, the incorporation of separate electrophysiological sessions at a time and place distinct from behavioral testing, made it unlikely that increased granule cell responsivity reflected either a general state of arousal or specific patterns of motor activity associated with the learning task.

Potentiation of evoked responses in the DG to PP stimulation was specifically related to training; this was observed in 2 separate groups of rats soon after the onset of training. Because the training phase preceded the control condition in one group of rats, and followed it in the other, the potentiation could not have been due to either the stimulation itself or to habituation and behavioral changes in the test sessions. Instead, the most likely causal factors for the physiological effect of training are those that most reliably affect behavior; namely, the positive contingencies between the tone, the response, and the food reward. Of course, other possibilities include simple presentations of tones; the temporal distribution of food; and acquisition of knowledge of the negative contingencies between food and incidental stimuli, irrelevant stimuli, ineffective behavioral responses, and the time elapsed since the previous trial. Unfortunately, the number of possible associations to be formed in almost every learning paradigm makes it difficult to control for all but one, and difficult to specify exactly which factor is most responsible for inducing potentiation.

A fundamental issue arising from the present results is the relation between training-induced potentiation and LTP. For the first time, training-induced potentiation and LTP were both assessed in the same subjects, and several points of similarity were observed. Both types of potentiation were present in every rat. In most cases, population spike amplitudes were increased over the entire range of intensities tested and the population

Table 1. Population spike amplitudes on the last days of each behavioral condition and on the pretetanic retest

\begin{tabular}{llllll} 
& & & Retest \\
\cline { 3 - 6 } & $\begin{array}{l}\text { Last day } \\
\text { free-feeding }\end{array}$ & $\begin{array}{l}\text { Last day } \\
\text { learning }\end{array}$ & Amplitude & $\begin{array}{l}\text { Inter- } \\
\text { val } \\
\text { (d) }\end{array}$ & Savings \\
\hline Group 1 & $100.4 \pm 10.4$ & $121.0 \pm 16.1$ & $124.0 \pm 7.2$ & 18 & $1.13 \pm 0.23$ \\
Group 2 & $90.3 \pm 8.1$ & $113.4 \pm 9.3$ & $108.1 \pm 17.7$ & 10 & $0.96 \pm 0.10$
\end{tabular}


spike thresholds werc reduced. The tetanic stimulation tended to induce more potentiation than did behavioral training, but it is worth noting that strong electrical stimulation was applied. In the present study, both groups were trained and showed comparable levels of training-induced potentiation before the tetanic stimulation was applied, and, therefore, the existence of an interaction between the 2 types of potentiation could not be tested. Perhaps a future study will reveal a negative interaction between the 2 types of potentiation, attributable to the mutual use of a finite set of modifiable synapses. Nevertheless, our results provide the first direct within-animal comparison of the 2 types of potentiation and reveal a remarkable similarity between them.

The ultimate question is whether LTP and training-induced potentiation share a common neural mechanism and are localized to the same cellular structures. Research on the mechanisms of LTP have localized it to either the presynaptic terminals (Dolphin et al., 1982) or the immediately postsynaptic region of the dendrites (Lynch and Baudry, 1984), and have shown potentiation in the PP-DG to be localized to the activated synapses (McNaughton and Barnes, 1977; McNaughton and Miller, 1984). Unfortunately, in the present study, as in almost all previous studies of training-induced potentiation, the locus of the cellular change could not be specified. Measurement of extracellular field potentials is well suited to long-term monitoring of synaptic efficacy, but cannot distinguish increased synaptic efficacy from other, nonsynaptic influences, such as changes in postsynaptic membrane conductance or alterations in the activity of extrinsic afferents. However, in one recent study of behaviorally induced potentiation in the PP-DG, in vitro measurements of hippocampal slices taken from rats reared in a complex environment revealed "experience-dependent synaptic alterations" localized to precisely the same regions of the cell as LTP (Green and Greenough, 1986).

In addition to these points of similarity between LTP and training-induced potentiation, an important distinction was revealed: training-induced potentiation displays a remarkable combination of stability and lability. Stability was demonstrated by almost complete savings over the intervals of 10 and $18 \mathrm{~d}$ between the end of training and the pretctanic tests. Lability, in the form of sensitivity to alterations in behavioral conditions, was revealed in the group switched from training to free-feeding during this same interval; the elevated population spike amplitudes returned to baseline values within $3 \mathrm{~d}$ of the reversal of behavioral conditions. Even though the mechanisms of the decline and spontaneous return of the training-induced potentiation are unknown, the pattern of results indicates that the potentiation did not dissipate, but was actively suppressed. In fact, there is a strong resemblance to the behavioral phenomena of conditioning, extinction, and spontaneous recovery.

Apparently, training-induced potentiation is stable as long as behavioral conditions remain constant; at the same time, it is labile in response to alterations in behavioral conditions. This conclusion is consistent with the effects of returning rats to a normal environment after rearing them in a complex one. Green and Greenough (1986) noted the absence of potentiation in hippocampal slices taken from such rats, contrasted with the clear potentiation present in slices taken from rats maintained in a complex environment. It is difficult to know whether this "experience-dependent synaptic alteration" was transient, as the authors suggest, or suppressed by the change in environment.

This combination of stability and lability is particularly ad- vantageous for a candidate mnemonic device, for it not only provides for enduring memories, but also permits behavioral adaptations to changes in environmental contingencies. In contrast, LTP suffers as a candidate mechanism by not being reversible (Teyler and DiScenna, 1986), and there is at least one study showing that LTP can induce a maladaptive behavioral inflexibility (McNaughton et al., 1986). In view of the greater adaptive value of training-induced potentiation, and its origins in natural, behaviorally relevant conditions, perhaps the most appropriate question is not whether training-induced potentiation shares a common mechanism with LTP, but whether LTP is a sufficiently accurate model of training-induced potentiation.

The observation of significant enhancement of population spike amplitudes in the DG of rats following acquisition of an appetitively motivated discriminated operant response parallels similar findings in rats and rabbits exposed to a variety of behavioral paradigms. Potentiation of granule cell responsivity is induced by classical conditioning of the nictitating membrane response in rabbits (Weisz et al., 1984) and by training in active avoidance in rats when the conditional stimulus is a tone (Rüthrich et al., 1982) or electrical stimulation of the PP (Matthies et al., 1986). Potentiation is also induced by exposure to a spatially complex environment, and has been measured in the DG of rats in vivo (Sharp ct al., 1985) and in vitro (Green and Greenough, 1986). In all but one of these studies (Weisz et al., 1984), the potentiation was measured in an environment separate from the one used for training. This implies the induction of more persistent changes in neural function, as would be expected with the formation of memory. Aside from the major finding of substantial and persistent increases in granule cell responsivity, perhaps the most significant feature of the "priorlearning" studies to date is the striking diversity of behavioral paradigms in which this effect can be found in a single region of the hippocampus. This, in turn, suggests that training-induced potentiation may represent a general process necessary for the encoding of many forms of new learning, as distinct from the storage of a specific experience in the form of modified synapses at the level of the DG.

Recently, Teyler and DiScenna (1986) have emphasized the capacity of the hippocampus to activate specific neocortical modules, and have proposed LTP as the mechanism for storing the addresses of the neocortical loci involved with information storage and retrieval. Training-induced potentiation may be a manifestation of this form of information processing in the hippocampus. Alternatively, the potentiation could reflect the operation of a "working memory" that is keeping track of behaviorally significant, but varying, information (Olton, 1983). Regardless of the exact mnemonic function of the hippocampus, it is now clear that enduring potentiation of communication between neurons occurs there during many forms of learning. Continued investigation of this phenomenon should contribute much to our understanding of the neural basis of learning and memory.

\section{References}

Berger, T. W. (1984) Long-term potentiation of hippocampal synaptic transmission affects rates of behavioral learning. Science 224: 627630.

Bliss, T. V. P., and A. R. Gardner-Medwin (1973) Long-lasting increases of synaptic transmission in the dentate area of the unanesthetized rabbit following stimulation of the perforant path. J. Physiol. (Lond.) 232: 356-374. 
Dolphin, A. C. (1985) Long-term potentiation at peripheral synapses. Trends Neurosci. 8: 376-378.

Dolphin, A. C., M. L. Errington, and T. V. P. Bliss (1982) Long-term potentiation of the perforant path in vivo is associated with increased glutamate release. Nature 279: 496-498.

Douglas, R. M., and G. V. Goddard (1975) Long-term potentiation of the perforant path-granule cell synapse in the rat hippocampus. Brain Res. 86: 205-215.

Durrant, J. E., H. R. Kingston, J. N. C. Sharp, and J. W. Kerr (1961) A New Analytic Geometry, Macmillan, Toronto, p. 30.

Green, E. J., and W. T. Greenough (1986) Altered synaptic transmission in dentate gyrus of rats reared in complex environments: Evidence from hippocampal slices maintained in vitro. J. Neurophysiol. 55: 739-750.

Lynch, G., and M. Baudry (1984) Biochemistry of memory: A new and specific hypothesis. Science 224: 1057-1063.

Lynch, G., C. Gall, and T. V. Dunwiddie (1978) Neuroplasticity in the hippocampal formation. In Maturation of the Nervous System: Progress in Brain Research, vol. 48, M. A. Corner, ed., pp. 113-128, Elsevier, Amsterdam.

Matthies, H., H. Ruethrich, T. Ott, H. K. Matthies, and R. Matthies (1986) Low frequency perforant path stimulation as a conditioned stimulus demonstrates correlations between long-term potentiation and learning. Physiol. Behav. 36: 811-821.

McNaughton, B. L., and C. A. Barnes (1977) Physiological identification and analysis of dentate granule cell responses to stimulation of the medial and lateral perforant pathways in the rat. J. Comp. Neurol. 175: 439-454.

McNaughton, B. L., C. A. Barnes, G. Rao, J. Baldwin, and M. Rasmussen (1986) Long-term enhancement of hippocampal synaptic transmission and the acquisition of spatial information. J. Neurosci. 6: 563-571
McNaughton, N., and J. J. Miller (1984) Medial septal projections to the dentate gyrus of the rat: Electrophysiological analysis of distribution and plasticity. Exp. Brain Res. 56: 243-256.

Morris, R., and M. Baker (1984) Does long-term potentiation/synaptic enhancement have anything to do with learning or memory? In Neuropsychology of Memory, L. R. Squire and N. Butters, eds., pp. 521535, Guilford, New York.

Olton, D. S. (1983) Memory functions and the hippocampus. In Neurobiology of the Hippocampus, W. Seifert, ed., pp. 335-373, Academic, New York.

Rüthrich, H., H. Matthies, and T. Ott (1982) Long-term changes in synaptic excitability of hippocampus cell populations as a result of training. In Neuronal Plasticity and Memory Formation, C. A. Marsan and H. Matthies, eds., pp. 589-594, Raven, New York.

Sharp, P. E., B. L. McNaughton, and C. A. Barnes (1985) Enhancement of hippocampal field potentials in rats exposed to a novel, complex environment. Brain Res. 339: 361-365.

Skelton, R. W., J. J. Miller, and A. G. Phillips (1983) Low frequency stimulation of the perforant path produces long-term potentiation in the dentate gyrus of unanesthetized rats. Can. J. Physiol. Pharmacol. 61: 83-89.

Skelton, R. W., J. J. Miller, and A. G. Phillips (1985) Long-term potentiation facilitates behavioral responding to single-pulse stimulation of the perforant path. Behav. Neurosci. 99: 603-620.

Teyler, T. J., and P. DiScenna (1984) Long-term potentiation as a candidate mnemonic device. Brain Res. Rev. 7: 15-28.

Teyler, T. J., and P. DiScenna (1986) The hippocampal memory indexing theory. Behav. Neurosci. 100: 147-154.

Weisz, D. G., G. A. Clark, and R. F. Thompson (1984) Increased responsivity of dentate granule cells during nictitating membrane response conditioning in rabbit. Behav. Brain Res. 12: 145-154. 